\title{
Fachbeitrag
}

Adelheid Mayer, Wolfgang Nikolaus Rappert, Maria Seissl

\section{Durch die Krise führen: Erfahrungen mit Corona- Maßnahmen an der Universitätsbibliothek der Universität Wien}

\section{Leading through the crisis: Experience with Corona measures at the University of Vienna Library}

http://doi.org/10.1515/abitech-2021-0006

Zusammenfassung: Die Ereignisse im März 2020 in Zusammenhang mit der Entwicklung der COVID-19 Erkrankungen zu einer Pandemie stellten Einrichtungen weltweit vor neue Herausforderungen. Der Beitrag reflektiert den Umgang mit der Krise an der Dienstleistungseinrichtung (DLE) Bibliotheks- und Archivwesen der Universität Wien und spannt den Bogen vom situativ angepassten Führungs- und Managementstil über den Umstieg auf einen rein virtuellen Betrieb bis zur Anpassung vieler Workflows an die geänderten Umstände. Ab Mai 2020 erfolgte die stufenweise Wiedereröffnung der physischen Bibliothek. Die Krise bedeutete insgesamt einen deutlichen Digitalisierungsschub für die Bibliothek, was auch eine Umfrage unter den Mitarbeiterinnen und Mitarbeitern am Ende des ersten Lockdowns belegt.

Schlagwörter: Bibliotheksmanagement, Coronakrise, Erfahrungsbericht

Abstract: In March 2020, the development of the COVID-19 virus situation into a full-blown pandemic presented institutions worldwide with unprecedented challenges. This article reflects the handling of the crisis by Vienna University Library and Archive Services staff, a service unit of Vienna University. Its response ranged from changes in leadership and management style to switching to a purely virtual mode of operation and necessary adaptations of workflows. From May 2020 onwards, the physical library reopened in several stages. Overall, the crisis brought a significant boost to digitization for the library, which was confirmed by a survey conducted among employees at the end of the first lockdown.

Keywords: Library management, Corona crisis, experience report

\section{Die Krise setzt ein}

Die Dienstleistungseinrichtung (DLE) Bibliotheks- und Archivwesen der Universität Wien stand Mitte März 2020 wie alle anderen Institutionen, Firmen und Betriebe vor einer Situation, für die Management und Belegschaft keine Erfahrungswerte hatten. Die Ereignisse überschlugen sich täglich, ja fast stündlich.

Wenn in der Managementtheorie situatives Führen als Managementansatz erläutert wird (was auch Bestandteil und Lehrinhalt des Curriculums des österreichischen Postgraduallehrgangs Library and Information Studies ist), war der März 2020 die Stunde der Bewährung in der Praxis. Grundsätzlich pflegt die Bibliotheksleitung der Universitätsbibliothek Wien einen deutlich kooperativen Führungsstil: Fragen des bibliothekarischen Alltags werden in abteilungsübergreifenden Arbeitsgruppen diskutiert, um alle Aspekte zu identifizieren und bestmögliche Ergebnisse zu erzielen. Situationen mit Zeitdruck lassen aber wenig Raum für ausgedehnte Besprechungen und tiefgehende Analysen, sodass die erste Phase des in Österreich Mitte März 2020 verfügten Lockdowns von einem unüblich direktiven Führungsstil geprägt sein musste. Folgende Stationen veranschaulichen das:

Während am Montag, den 9. März 2020, die Direktion noch verschiedene Routineaufgaben abarbeitete (etwa die Bearbeitung eines Dienstreiseantrags für Ende März, die Vorbereitung des Besuchs von fünf Kolleginnen und Kollegen aus Brasilien, Litauen, Kroatien und Spanien im Rahmen der für Mai geplanten Staff-Training-Week des Erasmus-Programms), hatten die permanent eingerichtete Arbeitsgruppe „Benutzung“ und das Systemteam bereits den Auftrag, die Konditionen für den Fall einer Bibliotheksschließung mit Stopp der Bestellfunktion, Aussetzen von Mahnroutinen und der flächendeckenden Verlängerung entliehener Werke zu planen. Am 9. und 10. März 


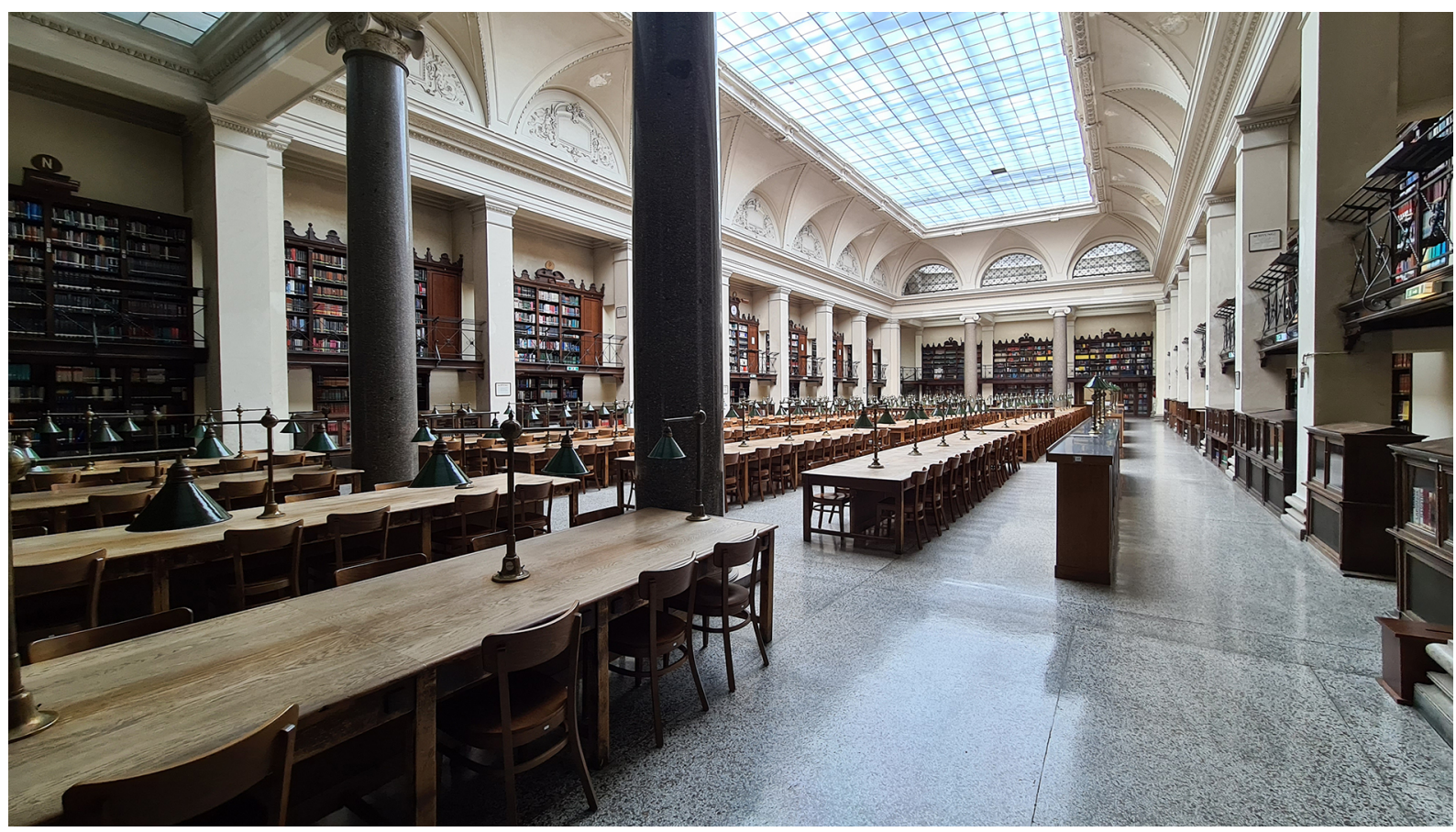

Abb. 1: Der mehr als 360 Personen Platz bietende Große Lesesaal der Universitätsbibliothek Wien im Lockdown (Foto: Universitätsbibliothek Wien)

konnte man parallel dazu am Benutzungsverhalten in den rund 40 Bibliotheksstandorten der Universitätsbibliothek Wien ablesen, dass bereits viele mit dem als Damoklesschwert über dem öffentlichen Leben schwebenden Lockdown rechneten: Lange Schlangen bildeten sich vor den Schaltern, um sich mit Literatur einzudecken.

Am Dienstag, den 10. März 2020, war klar: Das formale Einreichen des am Vortag avisierten Dienstreiseantrags erübrigt sich, die Erasmus-Planungen haben in der momentanen Situation keine Priorität, die Ergebnisse der Konsultationen von AG Benutzung und Systemteam werden dafür umso dringlicher. Erste Mitarbeiterinnen und Mitarbeiter machten sich Gedanken, welche Videokonferenztools als Ersatz für Dienstreisen zum Einsatz kommen könnten. An diesem Dienstag wurde auch die für Donnerstag, den 12.03.2020, anberaumte Vorstandssitzung der Vereinigung Österreichischer Bibliothekarinnen und Bibliothekare (VÖB) abgesagt und es wurde immer deutlicher: Eine Ausnahmesituation steht bevor.

Am Abend verfügte schließlich das Rektorat der Universität Wien die Umstellung auf Distance Learning mit unmittelbar einsetzender Wirkung ab 11.03.2020.

$\mathrm{Ab}$ Mittwoch waren die Universitätsgebäude folglich nur mehr für Mitarbeiterinnen und Mitarbeiter zugänglich; eine Möglichkeit zur Bücherrückgabe beim Portier des Hauptgebäudes wurde aktiviert, ebenso die Möglich- keit der Entleihung durch Mitarbeiterinnen und Mitarbeiter, die als solche nach wie vor Zugang zu den Universitätsgebäuden hatten.

Um Kontakte weiter zu reduzieren, gab das Rektorat am Freitag, den 13. März 2020, Anweisungen, wie der Dienstbetrieb im Back-Office-Bereich bis auf Weiteres zu organisieren sei: Für Home-Office qualifizierten sich Mitarbeiterinnen und Mitarbeiter, (1) die in den vergangenen zwei Wochen in Risikogebieten waren, (2) die in den vergangenen zwei Wochen Kontakt mit Covid-19-Erkrankten hatten, (3) die Betreuungspflichten aufgrund der Kindergarten- und Schulschließungen wahrnehmen mussten, (4) die zu den besonders gefährdeten Personen zu rechnen waren und (5) die mehr als eine Stunde öffentlich in die Arbeit zu fahren hatten. An diesem Freitag erbat die Bibliotheksdirektion von allen ihren über 400 Mitarbeiterinnen und Mitarbeitern um Selbstzuordnung zu diesen Kriterien und versuchte am Freitag und Samstag, die individuellen Home-Office-Situationen konkret festzulegen und jeder und jedem Rückmeldung zu geben.

Am Sonntag, den 15. März, waren diese Anstrengungen hinfällig, da die Regierung mit Wirksamkeit ab 16.03.2020 den allgemeinen Lockdown verfügte. Sonntagmittag setzte die Direktion alle Mitarbeiterinnen und Mitarbeiter per Mail in Kenntnis, dass die Arbeitsstätte ab Montag nicht mehr aufgesucht werden dürfe. Damit 
erreichte das Krisenmanagement einen Höhepunkt an direktivem Führungsstil.

\section{Arbeiten im Lockdown wird zur Erfahrungswirklichkeit}

Mit dem einsetzenden Lockdown waren die Kommunikationswege endgültig umzustellen. Auch wenn E-Mail-Korrespondenz als asynchrone Kommunikationsschiene gelten muss, war sie ein wichtiger Bestandteil der Kommunikation in der akuten Phase des startenden Lockdown, um die über 400 Mitarbeiterinnen und Mitarbeiter über laufende Entwicklungen $\mathrm{zu}$ informieren. Neben dieser Schiene etablierte sich aber auf Ebene der Leitung und der nachgeordneten Führungsebene als synchrones Kommunikationsmedium neben Telefonaten sehr rasch ein Videokonferenzsystem: Die Universität Wien setzt dabei auf MS Teams, und so trifft sich das Direktionsteam seit 24. März 2020 zur täglichen Koordination auf dieser Plattform. Nicht minder wichtig als asynchrones Arbeitsmittel war ein mit 26. März 2020 eingerichtetes universitätsinternes Wiki, in dem Maßnahmen des Notbetriebs der Bibliothek bibliotheksintern diskutiert und verschriftlicht wurden. Insbesondere Videokonferenzen und Wiki-Einträge boten den technischen Rahmen, in dem der in der Universitätsbibliothek Wien grundsätzlich bevorzugte kooperative Führungsstil wieder rasch aufgebaut werden konnte.

Alle weiteren Maßnahmen waren und sind dort abgebildet. Sehr rasch umgesetzt wurde etwa ein massiver Ausbau der ohnehin zahlreich vorhandenen elektronischen Ressourcen. Für Ressourcen, die üblicherweise nur für eine On-site-Nutzung lizenziert sind, konnte ein temporärer Fernzugriff mit den Anbietern verhandelt werden.

\section{Teilweise Rückkehr an den Arbeitsplatz und stufenweise Wiederaufnahme des physischen Bibliotheksbetriebs}

Mit der ersten, Anfang Mai 2020 einsetzenden Lockerungsstufe konnten Bibliotheksmitarbeiterinnen und -mitarbeiter die Arbeitsstätten grundsätzlich wieder betreten. Zwar war an eine Öffnung der Bibliotheken für einen regulären Präsenzbetrieb noch nicht $\mathrm{zu}$ denken, jedoch war ab 4. Mai 2020 ein Entleihbetrieb für Printressourcen in modifizierter Form wieder möglich: Systemseitig wurden in Freihandbereichen stehende Werke (die in den Fachbereichsbibliotheken der Universitätsbibliothek Wien übliche Aufstellungsart) über das System bestellbar gemacht. Seit diesem Zeitpunkt konnten Printressourcen wieder entlehnt werden. Die Öffnungszeiten zur Buchabholung und -rückgabe mussten in der Phase bis zum Sommer reduziert bleiben, da pro Standort die Mitarbeiterinnen und Mitarbeiter in Teams eingeteilt wurden, um Kontakte zu minimieren und nachverfolgbar zu machen. Flankierend wurden für alle Standorte und Schalterbereiche Plexiglasschutzwände, Desinfektionsmittel, Arbeitshandschuhe, MNS-Masken, Absperrbänder und Bodenmarkierungen zentral angeschafft und distribuiert, um für den Betrieb ein Höchstmaß an Schutz zu bieten.

Parallel zum wiedereinsetzenden physischen Betrieb baute die Universitätsbibliothek Wien ihre Digitalisierungsservices inklusive document delivery on demand aus: Da Scanmöglichkeiten an allen Standorten gegeben sind, kann eine Digitalisierung urheberrechtsfreier Werke sowie von Werken für Lehrveranstaltungen seither bei jeder Bibliothek angefragt werden.

Während die Front-Office-Tätigkeiten regelmäßige Anwesenheiten der Mitarbeiterinnen und Mitarbeiter vor Ort erforderlich machten, wurde der Back-Office-Bereich nach Maßgabe der Möglichkeiten jedenfalls teilweise im Home-Office belassen. Für Formal- und Sacherschließung erwies sich spätestens in Zeiten dieser Pandemie die Tatsache als hilfreich, dass das zum Einsatz kommende Bibliothekssystem Alma eine cloudbasierte Lösung ist und ohne lokale Installationen oder fix zugeordnete IP-Adressen auskommt. So konnten auch traditionell ortsgebundene Arbeiten wie Erwerbung, Formal- und SacherschlieBung ins Homeoffice verlegt werden.

Mit Sommer 2020 wurden die Lesesäle wieder geöffnet: Die Zahl der Sitzplätze musste freilich deutlich reduziert werden, um Abstandsregeln einhalten zu können. Für den Präsenzbetrieb galt es überdies, Contact-Tracing-Maßnahmen zu implementieren: Für sehr große Standorte wurde ein Sitzplatzreservierungstool eingeführt, mit dem Bibliotheksaufenthalte im Voraus online zu buchen sind. An kleineren Standorten wird eine Evidenz der Anwesenden auf unterschiedliche Weise sichergestellt: Manche Fachbereichsbibliotheken verbuchen für alle Besucherinnen und Besucher einen Garderobenschlüssel im System bzw. haben laminierte Karten erstellt, die mit einem im Bibliothekssystem verbuchbaren Barcode versehen sind. Andere Bibliotheken führen Aufzeichnungen auf Papier. Die erhobenen Daten werden nach spätestens vier Wochen datenschutzkonform vernichtet.

Im Verlauf des ersten Lockdowns sowie im Sommer erfolgte auch eine Umstellung auf virtuelle Schulungsmaß- 

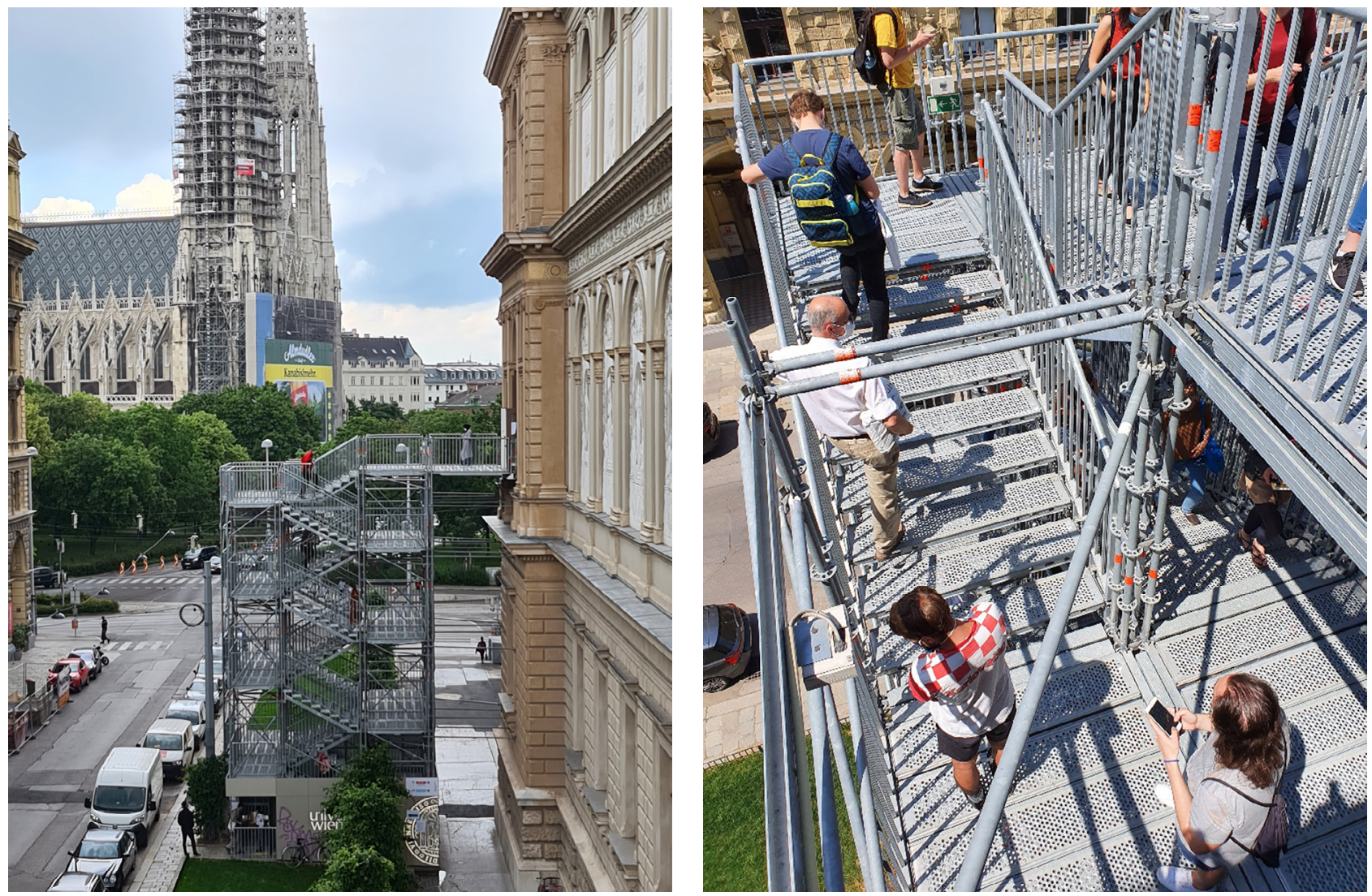

Abb. 2a, 2b: Seit 2016 sind am Universitätshauptgebäude der Universität Wien temporär Fluchttreppen angedockt, um eine rechtskonforme Entfluchtung des Großen Lesesaals zu ermöglichen. Diese bis zu einer (bereits in Planung befindlichen) General- und Funktionssanierung der Hauptbibliothek bestehenden Stiegen erweisen sich in der ersten Lockerungsstufe im Mai 2020 als sehr nützlich: Die Entlehnabteilung der Hauptbibliothek kann auf diesem Weg zugänglich gemacht werden, ohne das ganze Gebäude öffnen zu müssen. (Fotos: Universitätsbibliothek Wien)

nahmen, der Ausbau telefonischer Rechercheberatung sowie die Erstellung einer prototypischen virtuellen Tour durch die Hauptbibliothek, die auf weitere Bibliotheksstandorte ausgerollt werden soll. Die Erfahrungen mit virtuellen Besprechungsformaten haben sich sowohl in kleinen Teams als auch für größere Meetings bewährt: Die regelmäßig größte Zusammenkunft umfasst die gesamte zweite Führungsebene der Dienstleistungseinrichtung Bibliotheks- und Archivwesen, das sind rund 70 Personen. Bei diesen Zusammenkünften werden ganz regulär Tagesordnungen abgearbeitet und die Ergebnisse des Meetings protokolliert. Auch wenn diese Treffen gut funktionieren, ist das Fehlen des informellen Austauschs vor und nach den Zusammenkünften schwer zu kompensieren. Virtuelle Bewerbungsverfahren haben sich im Großen und Ganzen bewährt und werden voraussichtlich für Kandidatinnen und Kandidaten mit längerer Anreise als Option beibehalten.

\section{Die zweite Welle}

Leider haben sich die Zahlen in Österreich nach dem Sommer wie auch im übrigen Europa so entwickelt, dass es zu einem zweiten Lockdown kam. Dabei wurde zunächst ein „sanfter“ Lockdown verfügt, der für die Universitätsbibliothek Wien ab Anfang November bedeutete, die Präsenzbenutzung in den Lesesälen weitgehend einzustellen und nur nach Vereinbarung für nicht entleih- und nicht digitalisierbare Werke zuzulassen. Der Entleihbetrieb wurde im „sanften“ Lockdown allen Benutzerinnen und Benutzern ermöglicht. Da die Infektionszahlen nicht deutlich sanken, wurde Mitte November ein schärferer Lockdown verfügt. Die Universitätsbibliothek Wien blieb aber insofern geöffnet, als der Entleihbetrieb für Angehörige der Universität Wien aufrechterhalten werden konnte.

An dieser Stelle muss folgende Besonderheit erklärt werden: In Österreich sind die Universitäten bei COVIDNotmaßnahmenverordnungen grundsätzlich ausgenom- 


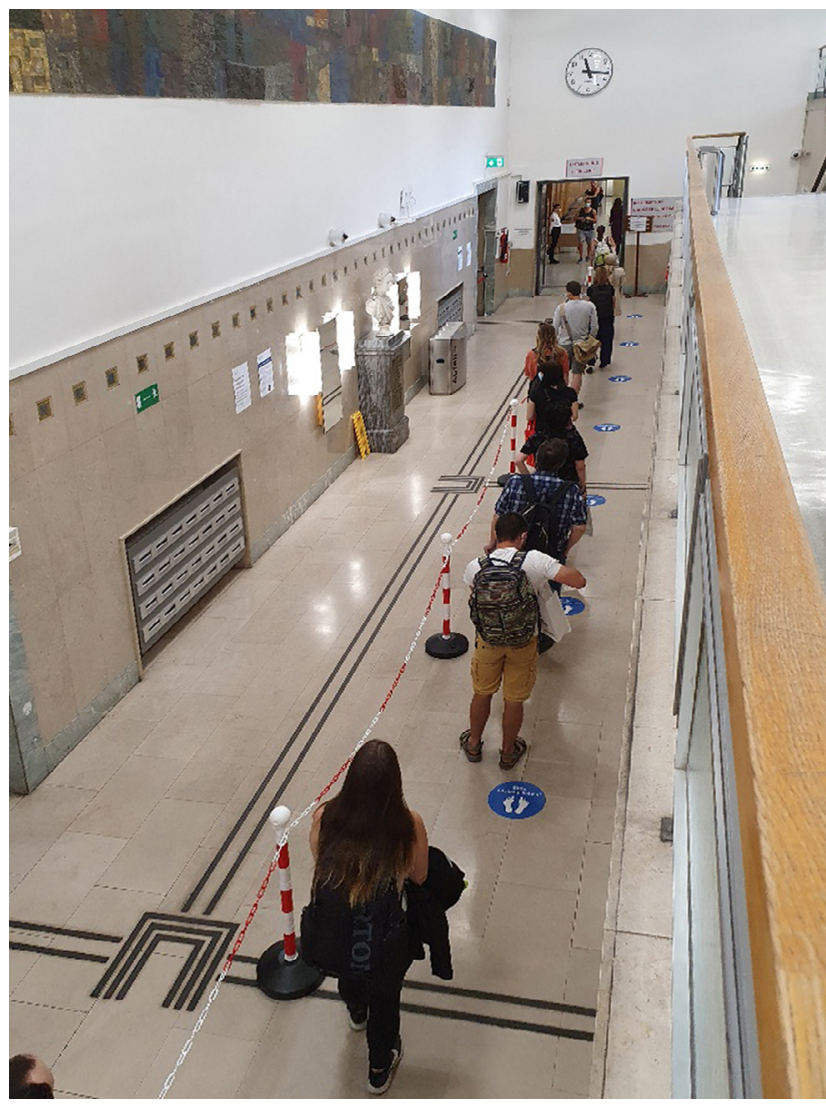

Abb. 3: Von der Fluchtstiege führt ein Einbahnsystem im Foyer der Hauptbibliothek zur Entlehnabteilung (Foto: Universitätsbibliothek Wien)

men und wurden in die Lage versetzt, die notwendigen Schritte - zwar in Absprache mit dem Wissenschaftsministerium, aber letztlich eigenständig im Rahmen ihrer Autonomie - selbst zu regeln. Wann immer Regierungsmitglieder, Expertinnen und Experten sowie Medienvertreterinnen und Medienvertreter davon gesprochen haben, dass aufgrund der jeweiligen Maßnahmen „Bibliotheken“ schließen müssen oder wieder aufsperren dürfen, waren und sind hierzulande die Österreichische Nationalbibliothek (die dem Bundesmuseengesetz unterliegt) sowie die Öffentlichen Büchereien gemeint. Nicht gemeint waren und sind die Universitätsbibliotheken, die als Organisationseinheiten ihrer jeweiligen Universität in den autonomen Gestaltungsbereich ihrer Universität fallen. Auch wenn die Universitäten als Institutionen grundsätzlich ausgenommen sind, gelten die COVID-Notmaßnahmen- und Lockerungsverordnungen nichtsdestotrotz auf individueller Ebene für die Bibliotheksbenutzerinnen und -benutzer. Die verfügten Ausgangssperren bedeuten daher zunächst, dass trotz eines eingeschränkten Biblio- theksbetriebes die Benutzerinnen und Benutzer gar nicht kommen dürfen.

Die COVID-Notmaßnahmen sehen allerdings auch auf der individuellen Ebene Ausnahmen von der strikten Ausgangssperre vor: Neben beruflichen Zwecken sind „Ausbildungszwecke“ als Ausnahme definiert. Die Verordnung wird so interpretiert, dass für die Anwendung dieser Bestimmung eine formal nachweisbare Ausbildungssituation vorauszusetzen ist. Diese wird im tertiären Bildungssektor in der Beziehung der eigenen Studierenden zur jeweiligen Universität oder Fachhochschule gesehen. So kam es zustande, dass im zweiten strikten Lockdown vom 17. November bis 6. Dezember zwar Studierende und Mitarbeiterinnen und Mitarbeiter der Universität Wien entleihen durften, andere Benutzergruppen allerdings nicht. Nach entsprechenden Konsultationen kam man überein, dass die Ausnahme der „Ausbildungszwecke“ doch auch über die Institutionsgrenzen hinaus Anwendung finden darf, so dass seit 1. Dezember 2020, also vor Ablauf des zweiten Lockdowns, Studierende anderer Bildungseinrichtungen ebenfalls für die Entleihung zugelassen werden konnten. All dies hat bibliotheksintern $\mathrm{zu}$ einer angeregten Diskussion geführt, wie es neben dem Versorgungsauftrag für Forschungs-, Lehr- und Studienzwecke der je eigenen Universitätsangehörigen um den öffentlichen Auftrag von Universitätsbibliotheken steht. Diese Diskussion ist an sich nicht neu, erhielt durch die Corona-Maßnahmen aber eine neue Brisanz und ist bis dato nicht abgeschlossen.

\section{Krisenbewåltigung: Ergebnisse einer Umfrage zu den
Auswirkungen des Lockdowns}

$\mathrm{Um}^{1}$ in Erfahrung zu bringen, wie die Mitarbeiterinnen und Mitarbeiter der Universitätsbibliothek und des Universitätsarchivs Wien die pandemiebedingte Krise subjektiv empfunden haben und welche Lehren daraus ihrer Meinung nach gezogen werden sollten, wurde Ende Mai von der Stabstelle Innovation eine interne Umfrage durchgeführt. Die hohe Zahl von über $50 \%$ aller Mitarbeiterinnen und Mitarbeitern, die die Umfrage beantwortet und abgeschlossen haben, überraschte und belegt die Notwendigkeit, den Mitarbeiterinnen und Mitarbeitern eine

1 Dieses Kapitel erscheint in abgeänderter Form unter dem Titel „Stress und Flexibilität“ von Adelheid Mayer in den VÖB-Mitteilungen $74,3 / 4$ (2020). 
Feedback-Möglichkeit zum Notfallmanagement und zur Krisenbewältigung zu geben.

Die Umstellung des Alltags durch den plötzlichen allgemeinen Lockdown wurde von der überwiegenden Mehrheit der Belegschaft relativ gelassen gesehen, nur etwas mehr als ein Drittel (36\%) empfanden ihn als stressig oder sehr stressig. Ebenso empfanden diejenigen, die aufgrund ihrer Arbeitsaufgaben zumindest im geringen Maße Homeoffice machen konnten (91\% der Antwortenden), dies überwiegend $(74 \%)$ als geringe oder gar keine Herausforderung. Die Arbeitsform Homeoffice wurde erstaunlich positiv angenommen. Der massive Wunsch, diese Arbeitsform auch nach dem Lockdown fortzuführen und auszubauen, zeigt sich nicht nur in der positiven Beantwortung der direkten Frage danach (83 \%), sondern wird auch in den freien Textfeldern der Umfrage immer wieder geäußert. Fast die Hälfte der Befragten (48 \%) schätzt diese Arbeitsform als gleich produktiv wie die Arbeit im Büro ein, ein weiteres Drittel (30\%) sogar als produktiver. Die rasche Umstellung auf Homeoffice hat auch eine Neuverteilung der Arbeitslast bewirkt - $34 \%$ gaben an weniger, $45 \%$ gleichviel und $21 \%$ mehr Arbeitsaufgaben gehabt zu haben.

Schwierigkeiten mit dem Homeoffice entstanden hauptsächlich durch technische Probleme bzw. mangelhafte technische Infrastruktur. Hier muss beachtet werden, dass die plötzliche Umstellung kaum Zeit ließ, sich privat mit der notwendigen Infrastruktur auszustatten, wenn sie noch nicht vorhanden war. Die Bemühungen der Beschaffung durch die DLE griffen erst allmählich, manche Produkte wie z. B. Headsets oder PC-Kameras waren auch im Online-Handel kaum noch zu bekommen. Eine weitere Herausforderung stellten für viele Mitarbeiterinnen und Mitarbeiter (22 \%) zusätzliche Betreuungspflichten dar, die neben beengten Wohn- und Arbeitsplatzverhältnissen als häufigste Gründe für Probleme im Homeoffice angeführt werden (Stichwort „Homeschooling“).

Die Beantwortung von Fragen im Bereich Arbeitsumfeld bezeugen den enormen Digitalisierungsschub, der in den Arbeitsweisen durch die Krise ausgelöst wurde. So gaben $70 \%$ der Befragten an, erstmals Kollaborations- und Videokonferenz-Software (z. B. MS Teams, Zoom, Skype) im beruflichen Kontext verwendet zu haben. Da digitale Meetings vielfach als produktiver empfunden werden, wurde der Wunsch geäußert, sie zumindest teilweise weiterhin anzuwenden, was rückwirkend betrachtet auch geschehen ist. Die Zusammenarbeit innerhalb der Teams wurde von drei Viertel als sehr gut oder gut empfunden, ebenso die Zusammenarbeit mit den Vorgesetzten.

Weit weniger rosig wurde das subjektive Empfinden der Arbeitssituation während des Lockdowns in einem
Freitextfeld mit Stichworten beschrieben. Die knappe Mehrheit der Antworten (33\%) beinhalten ausschließlich negativ konnotierte Gefühle und Eindrücke, 71 Antworten ( $29 \%$ ) hingegen rein positiv besetzte. 79 Einträge (= $32 \%)$ beinhalten Stichworte, die in beide Kategorien fallen und nur 7 (= $3 \%)$ sind als neutral (,wie immer“) einzustufen. Auffällig ist, dass bei den positiven Antworten am häufigsten die durch die Homeoffice-Situation entstandene Flexibilität und freie Zeiteinteilung ( $23 \%$ ) sowie einhergehend damit die Stressreduktion (22\%), die Möglichkeit auszuschlafen (14\%) und die höhere Produktivität (9\%) genannt wurden. An negativer Stelle stehen Stress und Überforderung (14\%), mangelnde Kommunikation v.a. mit Kolleginnen und Kollegen (13\%) sowie Vereinsamung (11\%).

Eine weitere Fragengruppe betraf das Management der Krise durch die Organisation Universität Wien bzw. DLE Bibliotheks- und Archivwesen. Rund $70 \%$ der Mitarbeiterinnen und Mitarbeiter haben sich von der Universitätsleitung in jeder Phase den Umständen entsprechend gut und ausreichend informiert gefühlt, bei der DLE-Leitung liegt dieser Prozentsatz sogar über $80 \%$. Ebenso empfand ein überwiegender Teil, dass Zuständigkeiten ausreichend kommuniziert wurden. Die Kommunikation des Lockdowns nach außen wurde überwiegend als gut eingestuft, die der virtuellen Services etwas weniger. Dass Arbeitsaufgaben gut verteilt worden wären, empfanden nur $38 \%$ der Antwortenden. Dies dürfte vor allem auf die Beschaffenheit der zu verteilenden Aufgaben zurückzuführen sein, wie mehrfach im freien Textfeld angemerkt wurde. So können und konnten Aufgaben aus der analogen Ausleihe kaum im Homeoffice erledigt werden.

Mit den Services der DLE beschäftigte sich die fünfte Fragengruppe. Virtuelle Services, allen voran Recherche-Tools und E-Ressourcen, funktionierten nach Ansicht der Mitarbeiterinnen und Mitarbeiter der DLE weiterhin gut, ebenso die Erwerbung von E-Books. Die Auskunftsdienste bekamen ein weniger gutes Zeugnis und die Forschungsunterstützenden Services sind intern offensichtlich noch immer vielfach unbekannt. Daraus resultierend soll nach Ansicht der Umfrage-Teilnehmerinnen und -Teilnehmer beim Ausbau der Services in den kommenden fünf Jahren der Schwerpunkt auf bessere Sichtbarkeit des Angebots der Universitätsbibliothek Wien gelegt werden, sowie virtuelle Schulungen und die Digitalisierung von Printbeständen vorangetrieben werden.

Eine überwiegende Mehrheit der Befragten ist der Ansicht, dass bei Beginn der COVID-19-Krise Notfallpläne nicht ausreichend vorhanden waren. Wurde bislang in Katastrophenszenarien meist vom digitalen Blackout ausgegangen, hat der analoge Lockdown die Institution kalt 
erwischt. Die Frage, ob für alle Mitarbeiterinnen und Mitarbeiter zugängliche Notfallpläne zu Katastrophen-Szenarien wie Feuer, Lockdown, Pandemie, Blackout, Wasserschäden usw. existieren sollten, wurde daher - wenig überraschend - überwältigend positiv bewertet. Darüber hinaus wird die Einrichtung eines Krisenstabes mit Vertreterinnen und Vertretern aus allen Bereichen ebenso als sinnvoll erachtet wie die Entwicklung von Workflows für Krisenfälle.

Doch um aus dieser Krise zu lernen, muss nach Ansicht der Befragten vor allem an der Kommunikation gearbeitet werden. Durch den Wegfall der zufälligen Kontakte und des informellen Austauschs ist offensichtlich geworden, dass viele Mitarbeiterinnen und Mitarbeiter am offiziellen Kommunikationsweg wenig partizipieren, da dieser vorrangig top-down stattfindet. Klar festgelegte Kommunikationsstrukturen und bessere, transparente Zuständigkeiten sowie bessere Aufgabenverteilung im Krisenfall werden ebenso als notwendig erachtet wie die raschere und konkretere Information nach innen und außen. Als weiterer Punkt, der aus der Krise mitgenommen werden soll, wird ein weiteres Mal der Ausbau der Möglichkeit im Homeoffice zu arbeiten, genannt.

Zusammenfassend kann gesagt werden, dass der Lockdown von den Mitarbeiterinnen und Mitarbeitern der Bibliothek und des Archivs angesichts der Umstände recht gut bewältigt wurde. Die virtuellen Services haben ohne Unterbrechung weiterhin funktioniert, und Arbeitsabläufe wurden rasch den geänderten Umständen angepasst. Quasi über Nacht wurde der Institution ein enormer Digitalisierungs-Schub verpasst. Die Änderung von Workflows und der Einsatz neuer Technologien, wie z. B. eines Platzreservierungssystems, gegen die oft jahrelanger interner Widerstand bestand, wurden plötzlich als sinnvoll und erstrebenswert erkannt. Der Einsatz moderner Kommunikations- und Kollaborations-Tools ist jetzt zur Selbstverständlichkeit geworden und viele Mitarbeiterinnen und Mitarbeiter haben die Vorteile des Homeoffice zu schätzen gelernt. Daneben muss aber das Ergebnis der Umfrage nicht zuletzt auch aufgrund der hohen Teilnahmezahl auch als Auftrag an das künftige strategische Handeln der DLE Bibliotheks- und Archivwesen verstanden werden. $\mathrm{Zu}$ nennen sind hier flexiblere Arbeitsformen, Verbesserung der internen und externen Kommunikation, Ausbau und bessere Sichtbarkeit der Services und nicht zuletzt eine umfassende Vorbereitung auf analoge und digitale Notfallsituationen.

\section{Zusammenfassung}

Bibliotheken sind eine zentrale Infrastruktur für Forschung, Lehre und Studium. Die Einschränkungen durch COVID-19 haben gezeigt, wie zentral. Während im ersten Lockdown der mangelnde Zugang zu gedruckter Literatur als größtes Hindernis wahrgenommen wurde, fehlt im zweiten Lockdown vor allem der Lernort Bibliothek (Lernplätze in den Lesesälen, gemeinsames Lernen in den Gruppenarbeitsräumen). Die Services der Bibliothek haben die Ortsgebundenheit hingegen überwunden: Der elektronische Bestand wurde massiv ausgebaut, Schulungen auf Onlinekurse umgestellt, Beratung per Telefon oder über E-Mail abgewickelt. Virtuelle Rundgänge geben Erstinformation und ersetzen Führungen, FAQs führen durch das Angebot, die Webseiten werden laufend aktualisiert. Systemseitig wurde alles unternommen, dass Benutzerinnen und Benutzer möglichst gar nicht vor Ort kommen müssen: automatische Verlängerungen, Digitalisierungs-Services (Bücher werden auf Bestellung als Scans verschickt), Document Delivery, Auskunft per Videochat, vermehrter Ankauf von elektronischer Literatur. Um Lesesaalbenutzung überhaupt möglich zu machen, wurde ein Reservierungstool implementiert, das hoffentlich bald wieder zur Anwendung kommen kann. Was auch nach der Krise bleiben wird: Online-Formate für Schulungen, Besprechungen und Meetings, das Reservierungstool für Leseplätze, gut ausgebaute Digitalisierungsservices, gut abgestimmte Öffnungszeiten, weitgehend digitalisierte Workflows, engagierte Mitarbeiterinnen und Mitarbeiter. Kurz: die Bibliothek. 


\section{Autoreninformationen}

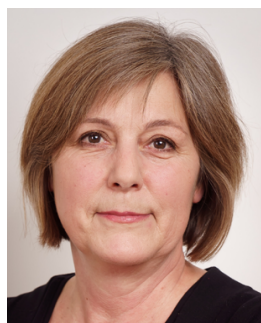

Mag. Adelheid Mayer, MSc

Leitung Innovation

Universität Wien

Universitätsbibliothek

Universitätsring 1

A-1010 Wien

adelheid.mayer@univie.ac.at

orcid.org/0000-0001-7923-5256

(C) Foto: derknopfdrücker

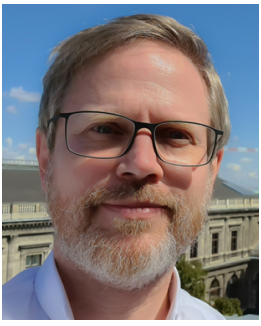

Dr. Wolfgang Nikolaus Rappert, MSc MAS

Stv. Leiter Bibliotheks- und Archivwesen

Universität Wien

Universitätsbibliothek

Universitätsring 1

A-1010 Wien

nikolaus.rappert@univie.ac.at

orcid.org/0000-0002-0860-0372

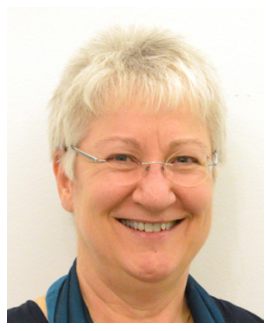

Mag. Maria Seissl

Leiterin Bibliotheks- und Archivwesen

Universität Wien

Universitätsring 1

A-1010 Wien

maria.seissl@univie.ac.at

orcid.org/0000-0001-5320-0223 